\title{
P60 Invasive Validation of Antares, a New Algorithm to Calculate Central Blood Pressure from Oscillometric Upper Arm Pulse Waves
}

Marcus Doerr ${ }^{1,2}$, Stefan Richter ${ }^{3}$, Siegfried Eckert ${ }^{4}$, Marc-Alexander Ohlow ${ }^{3}$, Fabian Hammer ${ }^{1}$, Astrid Hummel ${ }^{1}$, Vivien Dornberger ${ }^{1}$, Elisabeth Genzel ${ }^{1}$, Johannes Baulmann ${ }^{5,6, *}$

${ }^{1}$ Department of Internal Medicine B, University Medicine Greifswald, Germany

${ }^{2}$ German Centre for Cardiovascular Research (DZHK), partner site Greifswald, Germany

${ }^{3} Z$ entralklinik Bad Berka GmbH, Herzzentrum, Department of Cardiology, Germany

${ }^{4}$ Klinik für Allgemeine und Interventionelle Kardiologie/Angiologie, Universitätsklinik der Ruhr-Universität Bochum, Bad Oeynhausen, Germany

${ }^{5}$ Medizinische Universität Graz, Austria

${ }^{6}$ European Prevention Center, Düsseldorf, Germany

\section{ABSTRACT}

Background: Antares is an algorithm for pulse wave analysis (PWA) by oscillometric blood pressure monitors in order to estimate central (aortic) blood pressure (cBP). Antares aims to enable brachial cuff-based blood pressure (BP) monitors to be type II-devices, determining absolute $\mathrm{cBP}$ values independently of potential peripheral $\mathrm{BP}$ inaccuracies. The present study is an invasive validation of the Antares algorithm.

Methods: We followed entirely the 2017 ARTERY protocol for validation of non-invasive cBP devices, the 2013 ANSI/AAMI/ ISO 81060-2 and 2018 AAMI/ESH/ISO validation standard protocols. In total, 191 patients undergoing cardiac catheterization were included, of which 145 patients entered analysis. Invasive cBP recordings were prospectively compared to simultaneous non-invasive $\mathrm{cBP}$ estimations using Antares, integrated into an oscillometric BP monitor. Antares analyses pulse waves during deflation of the cuff (patent of Redwave Medical GmbH). Generally speaking, pulse waves of a normal oscillometric BP measurement are taken for PWA with no need for altering standard BP pump operation.

Results: Mean difference between invasive and non-invasively estimated systolic cBP was $0.71 \mathrm{mmHg}$ with standard deviation of $5.95 \mathrm{mmHg}$, fulfilling the highest validation criteria. Bland-Altman plot reveals good limits of agreement.

Conclusion: Antares is the first algorithm for estimation of cBP that entirely fulfills the 2017 ARTERY and AAMI/ESH/ISO validation protocols including criteria for high accuracy devices. The Antares algorithm turns the oscillometric upper arm blood pressure monitor into a type II-device for estimation of true $\mathrm{cBP}$. Integration of Antares into BP monitors could enable the measurement of PWA parameters in virtually every practice in future.

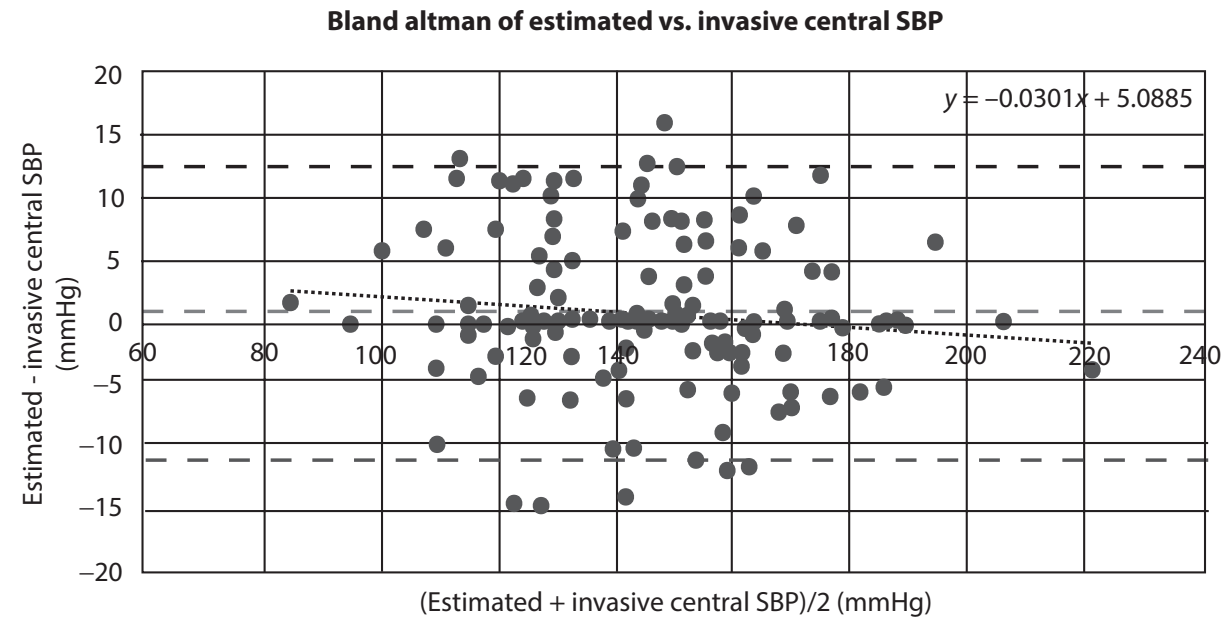

(c) 2019 Association for Research into Arterial Structure and Physiology. Publishing services by Atlantis Press International B.V. This is an open access article distributed under the CC BY-NC 4.0 license (http://creativecommons.org/licenses/by-nc/4.0/). 